Research Article

\title{
Field Equations and Lagrangian of the Kaluza Energy-Momentum Tensor
}

\author{
L. L. Williams \\ Konfluence Research Institute, Manitou Springs, Colorado, USA \\ Correspondence should be addressed to L. L. Williams; willi@konfluence.org
}

Received 23 January 2020; Revised 26 March 2020; Accepted 4 April 2020; Published 4 May 2020

Academic Editor: Soheil Salahshour

Copyright @ 2020 L. L. Williams. This is an open access article distributed under the Creative Commons Attribution License, which permits unrestricted use, distribution, and reproduction in any medium, provided the original work is properly cited.

\begin{abstract}
We provide an analysis and statement of the source term in the classical Kaluza field equations, by considering the 5-dimensional (5D) energy-momentum tensor corresponding to the 5D geodesic hypothesis that is typically presumed in the Kaluza theory. By providing the 5D matter Lagrangian, this work completes a Lagrangian analysis of the classical Kaluza theory that began by establishing the proper form of the unique Kaluza field Lagrangian. This work considers the transformation properties necessitated by general covariance of the Kaluza source terms, to establish the correct form for the source terms in the field equations, and to establish the 5D matter Lagrangian that corresponds to the 5D geodesic hypothesis. In addition to the effects of a scalar field expected from other scalar-tensor or scalar-electromagnetic theories, a peculiar Kaluza coupling coefficient arises for charged matter which acts to vary the source strengths in ways unknown to conventional physics. We briefly evaluate the implied modifications to source terms in the field equations. We find an ADM-like neutralization of gravity at high specific charge states and a saturation in field strength at high specific charge-to-mass ratios.
\end{abstract}

\section{Introduction and Previous Results}

Kaluza [1] recognized that the tensor gravitational potential of general relativity, and the vector potential of electromagnetism, could be understood as components of a 5-dimensional (5D) tensor gravitational potential. This unification implies an additional 15th component that behaves as a scalar under $4 \mathrm{D}$ transformations. Kaluza originally set the Kaluza scalar field (KSF) to a constant; indeed, the $4 \mathrm{D}$ limit of the Kaluza ansatz occurs when the KSF goes to 1 . The full field equations including the KSF were developed over succeeding decades, and by multiple independent research groups, e.g., [2-6]. Ref. [7] provides a history of the various groups, led by Scherrer in Switzerland, Lichnerowicz in France, Jordan in Germany, and Dicke at Princeton. We can also add associates of Einstein at Princeton in the 1930s.

Klein proposed a compact, microscopic interpretation of the 5th dimension [8], which has informed "Kaluza-Klein" theories since. There is no logical necessity to the microscopic assumption [6], and it raises as many difficulties as it resolves. We consider here instead the purely classical theory, with the 5th dimension allowed to be fully open and macroscopic.

The Kaluza ansatz is to write the Einstein equations in 5 dimensions:

$$
\tilde{G}_{a b}=\frac{8 \pi \tilde{G}}{c^{4}} \tilde{T}_{a b}
$$

where $\tilde{G}_{a b}$ is a $5 \mathrm{D}$ Einstein tensor for a $5 \mathrm{D}$ metric $\tilde{g}_{a b}$, $\tilde{G}$ is a $5 \mathrm{D}$ gravitational constant, $c$ is the speed of light, and $\tilde{T}_{a b}$ is a $5 \mathrm{D}$ energy-momentum source term. Small roman indices range over the 5 dimensions. Kaluza enforced the lack of an apparent fifth dimension with the "cylinder condition," $\partial \tilde{g}_{a b} / \partial x^{5}=0$, meaning that no fields depend on the fifth coordinate. Without this condition, many more degrees of freedom result, and there is no clear mapping to familiar physics. 
The components of the 5D metric $\tilde{g}_{a b}$ are given in terms of the $4 \mathrm{D}$ metric $g_{\mu \nu}$, the electromagnetic covariant 4 -vector potential $A_{\mu}$, and a scalar field $\phi$ :

$$
\begin{aligned}
& \tilde{g}_{\mu \nu}=g_{\mu \nu}+\phi^{2} k^{2} A_{\mu} A_{v}, \\
& \tilde{g}_{5 v}=\phi^{2} k A_{v}, \\
& \tilde{g}_{55}=\phi^{2} .
\end{aligned}
$$

where Greek indices range over the 4 dimensions of spacetime and the index 5 denotes the fifth dimension. We allow the sign of $\tilde{g}_{55}$ to be unspecified for now. Because $\tilde{g}_{a b} \tilde{g}^{b c}=\delta_{a}^{c}$, the inverse metric is given by:

$$
\begin{aligned}
& \tilde{g}^{\mu v}=g^{\mu \nu}, \\
& \tilde{g}^{5 v}=-k A^{v}, \\
& \tilde{g}^{55}=k^{2} A^{2}+\frac{1}{\phi^{2}} .
\end{aligned}
$$

The constant $k$ is the characteristic electro-gravitic scale parameter of the Kaluza theory, combining the gravitational constant $G$ and either the permittivity of free space, $\varepsilon_{0}$, or the permeability of free space, $\mu_{0}$ :

$$
k c \equiv \sqrt{16 \pi G \varepsilon_{0}}=\sqrt{\frac{16 \pi G}{\mu_{0} c^{2}}} \simeq 1.7 \times 10^{-10} \mathrm{C} / \mathrm{kg} .
$$

$k$ is typically written in CGS units in other references, so the electric dependence is somewhat obscured. Here we use MKS units, which makes the electrical dependence explicit. There are no free parameters in the Kaluza theory, and $k$ is the only characteristic constant. $k$ is a classical quantity, closely related to the ADM mass [9]. Note that $k A_{\mu}$ is unitless.

The $4 \mathrm{D}$ field equations are contained in the 5D Einstein tensor $\tilde{G}_{a b}$ : the Einstein equations with electromagnetic and scalar field energy-momentum are contained in $\tilde{G}_{\mu \nu}$; the Maxwell equations modified by a scalar field are in $\tilde{G}_{5 \mu}$; and $\tilde{G}_{55}$ provides an equation for $\phi$.

To resolve apparent contradictions among expressions for the field equations found in the English language literature, $\tilde{G}_{a b}$ was evaluated using tensor algebra software [10], and the associated Kaluza Lagrangian was established from the 5D Hilbert Lagrangian density to be

$$
\mathscr{L}=g^{1 / 2}\left[\frac{c^{4} \phi}{16 \pi G} g^{\alpha \beta} R_{\alpha \beta}-\frac{\phi^{3}}{4 \mu_{0}} g^{\alpha \mu} g^{\beta v} F_{\alpha \beta} F_{\mu \nu}\right],
$$

where $R_{\mu \nu}$ is the Ricci tensor, $F_{\mu \nu} \equiv \partial_{\mu} A_{\nu}-\partial_{\nu} A_{\mu}$ is the electromagnetic field strength tensor, $g$ is the determinant of $g_{\mu \nu}$, and the gravitational field equations are obtained from variation with respect to $g^{\mu \nu}$.

A review of the literature based on [10] shows that few authors recover this Lagrangian, and the literature is littered with algebraic errors in either the $5 \mathrm{D}$ curvature tensor $\tilde{R}_{a b}$ or the 5D Einstein tensor $\tilde{G}_{a b}$. Only Ref. [4] was found to accurately capture the curvature and Einstein tensor components, as well as the Lagrangian (5). Ref. [5] provides the correct Einstein tensor components and Lagrangian but makes a few errors in the curvature tensors. Refs. $[3,6]$ have the correct curvature tensors.

The expression (5) follows uniquely from a consideration of the $5 \mathrm{D}$ Hilbert Lagrangian, $\tilde{g}^{1 / 2} \tilde{R}$. The form of (5) is quite unusual, because it depends only algebraically on the KSF. Even so, full scalar field dynamics are still obtained in the theory, as we will see. Still, other authors reflexively add an additional standard scalar field kinetic term $\propto\left(\partial_{\mu} \phi\right)\left(\partial^{\mu} \phi\right)$, as in the Brans-Dicke theory [11]; but this degree of freedom is not naturally in the Kaluza theory once the cylinder condition is imposed.

It is true that a conformal transformation can remove the scalar field from the gravitational term in (5), but it comes at the expense of adding a scalar field kinetic term to the Lagrangian. The frame with a scalar field is the "Jordan frame," and the conformally transformed frame with the scalar field kinetic term is the "Einstein frame." The conformal transformation does not change the physics, however, and particles still move on geodesics of the Jordan frame, not the Einstein frame [12]. The electromagnetic term in (5) is invariant under a conformal transformation [5].

Note that the KSF acts both as a variable gravitational constant, as in [11], and as a variable dielectric constant, as in [13]. This makes the theory quite unique among scalartensor theories, and among scalar-electromagnetic theories. The textbook, $4 \mathrm{D}$ limit is $\phi \longrightarrow 1$.

In this work, we provide an analysis of the source term in (1) and thereby complete the Kaluza field equations. We find that $5 \mathrm{D}$ covariance constrains the form of the source term. We will then go on to consider implications for these source terms in the electromagnetic, scalar, and gravitational field equations.

\section{Identification of Electric Charge}

In addition to the correspondence in the field equations, Kaluza [1] also recognized that the 5D geodesic equation contained the $4 \mathrm{D}$ geodesic equation plus the Lorentz force of electromagnetism, and identified electric charge with motion along the fifth coordinate. However, many authors, including Kaluza, considered weak charge states only, to avoid the apparent modifications of the field equations that occur in highly charged systems. An exact treatment for all charge states will be provided here.

Various authors make different identifications of electric charge in terms of the Kaluza theory parameters. The first step is to precisely identify electric charge in terms of the Kaluza theory parameters and relate it to choices made by other authors. Therefore we start like Kaluza, with the 5D geodesic equation for a particle with $5 \mathrm{D}$ proper velocity $\tilde{U}^{a}$ :

$$
\frac{d \tilde{U}^{a}}{d s}+\tilde{\Gamma}_{b c}^{a} \tilde{U}^{b} \tilde{U}^{c}=0
$$


where

$$
\begin{aligned}
\tilde{U}^{a} & \equiv \frac{d x^{a}}{d s}, \\
d s^{2} & \equiv \tilde{g}_{a b} d x^{a} d x^{b} .
\end{aligned}
$$

From (2), it follows that the 4D and 5D length elements are related:

$$
a^{2} d s^{2}=g_{\mu v} d x^{\mu} d x^{v}+\phi^{2}\left(d x^{5}+k A_{v} d x^{v}\right)^{2},
$$

where $a$ is an undetermined constant.

Consider first the equation for the fifth component of $\tilde{U}^{a}$. Since $\partial_{5} \tilde{g}_{a b}=0$, the covariant component $\tilde{U}_{5}$ is a constant of the motion:

$$
\tilde{U}_{5}=\tilde{g}_{5 b} \tilde{U}^{b}=\phi^{2}\left(\tilde{U}^{5}+k A_{v} \tilde{U}^{v}\right)=\text { constant } .
$$

It is important to note that the cylinder condition is far from simplistic. It implies a meaningful and nontrivial constant of the motion.

Joining (8) and (9) provides an expression that relates $d s^{2}$ to the $4 \mathrm{D}$ length element $c^{2} d \tau^{2} \equiv g_{\mu \nu} d x^{\mu} d x^{\nu}$, and this expression depends only on the magnitude of the invariant $\tilde{U}_{5}$ and the scalar field

$$
c^{2}\left(\frac{d \tau}{d s}\right)^{2}=a^{2}-\frac{\tilde{U}_{5}^{2}}{\phi^{2}}
$$

The relations (8), (9), and (10) are common in the Kaluza literature.

Now we evaluate the spacetime components of the 5D geodesic equation (6), for the metric (2) and using (9). The various $\tilde{\Gamma}_{b c}^{a}$ for (2) are tabulated in Ref. [10]. We find

$$
\frac{d \tilde{U}^{v}}{d s}+\Gamma_{\alpha \beta}^{v} \tilde{U}^{\alpha} \tilde{U}^{\beta}+k \tilde{U}_{5} \tilde{U}^{\alpha} g^{\nu \mu} F_{\alpha \mu}-g^{v \alpha} \phi\left(\partial_{\alpha} \phi\right)\left(\frac{\tilde{U}_{5}}{\phi^{2}}\right)^{2}=0 .
$$

This equation is obtained by Refs. [4, 5, 14]. The $4 \mathrm{D}$ gravitational, electromagnetic, and scalar force terms are cleanly separated. Its remarkable simplicity results from a grouping of terms according to $\tilde{U}_{5}$, instead of $\tilde{U}^{5}$.

The term in $F_{\mu \nu}$ obviously must be identified with the Lorentz force, and Ref. [14] stops here to assign the coefficient in (11) as electric charge. We continue instead, because (11) does not yet involve the 4D proper velocity.

Therefore, we use (10) to transform (11):

$$
\frac{d U^{\nu}}{d s}+\Gamma_{\alpha \beta}^{v} \tilde{U}^{\alpha} U^{\beta}=k \tilde{U}_{5} g^{\nu \mu} F_{\mu \alpha} U^{\alpha}+\tilde{U}_{5} U_{5}\left(\partial_{\alpha} \ln \phi\right)\left[g^{\nu \alpha}-\frac{U^{\nu} U^{\alpha}}{c^{2}}\right],
$$

where the $4 \mathrm{D}$ proper velocity of a particle is

$$
U^{\mu} \equiv \frac{d x^{\mu}}{d \tau},
$$

and where

$$
U_{5} \equiv U^{5}+k A_{\mu} U^{\mu}
$$

We choose to identify electric charge with the coefficient in (12). Therefore, we identify the electric charge $Q$ of a body of rest mass $m_{0}$ :

$$
k c \tilde{U}_{5} \longrightarrow \frac{Q}{m_{0}} .
$$

The identification (15) and the 4D equation of motion (12) are also obtained by Refs. [4] and [14]. While there is some discomfort to still having the fields enter the definition of electric charge (9), their grouping in the equations of motion makes the identification unavoidable.

Note that this is a classical theory, and the ratio (15) does not strictly apply to elementary charges. Instead, (15) is the ratio of the electric charge to mass of a macroscopic volume of charged, massive fluid. The differential volume elements are assumed to be composed of a macroscopic amount of charge carriers, and (15) can be considered specific charge. Therefore, charge density $\sigma=\rho k c \tilde{U}_{5}$, and the electromagnetic 4-current $J^{v}=\rho k c \tilde{U}_{5}\left(d x^{v} / d t\right)$.

There are two pieces to the scalar force in (12), and this is indeed the form expected for a scalar force [15]. Therefore, the term (10) in $d \tau / d s$ is critical to a proper representation of the $4 \mathrm{D}$ scalar force, although other references overlook the second term in the scalar force.

The scalar force couples to electrically charged bodies, and it does so quadratic in the electric charge. This means the scalar force depends only on the magnitude of electric charge, not its sign. The scalar force vanishes for neutral bodies, and the field equations and equations of motion look very similar to their $4 \mathrm{D}$ counterparts in the neutral limit, since $d \tau / d s \longrightarrow a / c$ and $\tilde{U}_{5} \longrightarrow 0$. The radically different nature of the scalar field coupling makes the KSF unique among other scalar-tensor theories that imagine the scalar field coupling to neutral matter mass-energy, like gravity itself.

Because $\tilde{U}_{5}$ includes a dependence on $\tilde{U}^{\mu}$, the theory predicts an induced electric charge $Q_{i}=m_{0} c k^{2} A_{\mu} \tilde{U}^{\mu}$ for apparently neutral bodies moving in an electromagnetic field. For example, it seems to indicate deflection of neutral bodies in magnetic fields, under particular circumstances. By the same token, it appears that the induced electric charge could also allow neutral bodies to couple to the scalar force.

Let us write an alternative form for (10):

$$
\left(\frac{d s}{d \tau}\right)^{2}=\frac{c^{2}}{a^{2}-\tilde{U}_{5}^{2} / \phi^{2}}
$$


Now put together (9), (14), and (16) to find

$$
U_{5}=\frac{c \tilde{U}_{5} / \phi^{2}}{\sqrt{a^{2}-\tilde{U}_{5}^{2} / \phi^{2}}} .
$$

Expressions (10), (16), and (17) enter as effective coupling coefficients in the field equations, and they behave differently depending on whether $Q \ll m_{0} k c$ or $Q \gg m_{0} k c$. The coefficient (10) can be identified as an effective mass $[4,14]$.

\section{5D Energy-Momentum Tensor}

Kaluza also originally considered a 5D energy-momentum tensor for dust, and subsequent authors have as well. They typically consider the form $\tilde{T}_{a b} \propto \rho \tilde{U}_{a} \tilde{U}_{b}$, which is only covariant if the density is referred to a comoving frame. Additionally, some treatments were not exact or were restricted to weak charge densities. Here we provide a careful consideration of the transformation properties necessary to construct a proper energy-momentum tensor.

Like other authors, we consider 5D dust, consistent with (6). This choice for the form of the energy-momentum tensor is necessitated by our previous considerations of the geodesic equation, because the geodesic equation (6) follows from relativistic conservation of energy applied to dust. By dust, we mean massive fluid particles, with no internal energy. It is an ensemble of particles. This dust is electrically charged, with no quantized charge carriers, but a specific charge per unit mass.

We can merely write down the form of a covariant dust energy-momentum tensor, based on standard results [16]:

$$
\tilde{T}_{a b}=\frac{\tilde{\rho}}{\tilde{g}^{1 / 2}} \frac{\tilde{U}_{a} \tilde{U}_{b}}{(d t / d s)},
$$

where $\tilde{U}_{a}=\tilde{g}_{a b} \tilde{U}^{b}$. The time coordinate is picked out as independent against the other 4 coordinates.

By construction, $\tilde{\rho}$ is a $4 \mathrm{D}$ density, with units of mass per volume per unit fifth coordinate. An integration over the 5 th coordinate is implied whenever an invariant integral is taken over the spatial coordinates, $\propto \int \tilde{\rho} g^{1 / 2} d^{3} x \phi d x^{5}$. Because of the cylinder condition, the $x^{5}$ integral has no functional dependence and can be set to 1 . Therefore we can collapse $\tilde{\rho} \longrightarrow \rho$, with units of mass per volume as usual.

The factor $\tilde{g}^{1 / 2}$ in (18) is crucial to the $5 \mathrm{D}$ covariance of $\tilde{T}_{a b}$, thereby matching the 5D covariance of $\tilde{G}_{a b}$ in (1) and making it a tensor. There is some confusion, because a standard description for dust, for example in cosmology, references the fluid density to a comoving frame, and the factor $g^{1 / 2}$ is ignored. Yet its presence here affects the field equations because, as shown in Ref. [10], $\tilde{g}^{1 / 2}=\phi g^{1 / 2}$.

Recall that the energy-momentum tensor can be defined in terms of a variation of the matter action:

$$
\tilde{T}_{a b}=\frac{-2}{\tilde{g}^{1 / 2}} \frac{\delta S_{M}}{\delta \tilde{g}^{a b}},
$$

where $S_{M}=\int \mathscr{L}_{M} d^{4} x$. Therefore, we can write the matter Lagrangian density corresponding to (18) and expand it using the 5D metric (3):

$$
\begin{aligned}
\mathscr{L}_{M} & =g^{1 / 2} \hat{\mathscr{L}}_{M}=-\frac{1}{2} \tilde{\rho} c \frac{\tilde{U}_{a} \tilde{U}_{b}}{(d t / d s)} \tilde{g}^{a b} \\
& =-\frac{1}{2} \rho c \frac{d s}{d t}\left[g^{\mu \nu} \tilde{U}_{\mu} \tilde{U}_{v}-2 k A^{v} \tilde{U}_{5} \tilde{U}_{v}+\left(k^{2} g^{\mu v} A_{\mu} A_{v}+1 / \phi^{2}\right) \tilde{U}_{5}^{2}\right] .
\end{aligned}
$$

The matter Lagrangian (20) complements the field Lagrangian (5) to provide a full description of the 5D theory. While the field equations are more easily obtained from the field Lagrangian than from direct evaluation of the curvature tensors, the source terms can be obtained with equal effort from the matter Lagrangian or from the 5D energy-momentum tensor.

Having now established the necessary form of the source term from 5D covariance principles, we turn to its implications for modifications to the Maxwell and Einstein equations for charged matter.

\section{Field Equations with Sources}

Many Kaluza references consider only the vacuum equations, consider an additional term in the field Lagrangian, restrict attention to low charge densities, or consider an energymomentum tensor valid only in a comoving frame. Here we present new results that describe the modifications to the source terms in the Einstein gravitational field equations, and Maxwell electromagnetic field equations, that arise inescapably from a 5D covariant source term (18) that is consistent with the 5D geodesic equation (6).

4.1. Gravitational Field Equations. We consider first the Kaluza gravitational field equations with covariant derivative $\nabla_{\mu}$. There are modifications from the KSF expected from the vacuum equations, but also new, unexpected effects from the matter source terms.

$$
\begin{aligned}
G_{\mu \nu}= & \phi^{-1} T_{\mu \nu}^{\phi}+\frac{8 \pi G}{\mu_{0} c^{4}} \phi^{2} T_{\mu \nu}^{\mathrm{EM}} \\
& +\frac{8 \pi G}{c^{3} \phi} \frac{d \tau}{d s} \frac{\rho}{g^{1 / 2}} g_{\mu \alpha} \frac{d x^{\alpha}}{d t} g_{\nu \beta} \frac{d x^{\beta}}{d \tau},
\end{aligned}
$$

where $d \tau / d s$ is given by (10), and where

$$
T_{\mu \nu}^{\phi} \equiv \nabla_{\mu} \nabla_{\nu} \phi-g_{\mu \nu} \nabla_{\alpha} \nabla^{\alpha} \phi
$$

is the KSF energy-momentum tensor, and where

$$
T_{\mu \nu}^{\mathrm{EM}} \equiv g^{\alpha \beta} F_{\mu \alpha} F_{\nu \beta}-\frac{1}{4} g_{\mu \nu} F_{\alpha \beta} F^{\alpha \beta},
$$

is the electromagnetic energy-momentum tensor. There is an effective variable gravitational constant, $G / \phi$, in the matter term of (21), as expected from the Brans-Dicke theory. But the term $c d \tau / d s$ acts as a Kaluza coupling coefficient that is not anticipated in standard scalar-tensor gravity. 
Both the KSF and the electromagnetic (EM) field energymomentum in (21) contribute to curvature of the 4D metric. The KSF coupling is algebraically different between matter, EM field, and scalar field-a departure from standard scalar-tensor theory.

The KSF energy-momentum tensor is dimensionless in (21); there is no explicit coupling constant. This is different than the standard scalar-tensor theory. Here, the KSF energy-momentum is more akin to a cosmological constant, which also is without any scaling parameters.

The trace of the field equations (21) yield

$$
R=\frac{3}{\phi} \nabla_{\alpha} \nabla^{\alpha} \phi+\frac{8 \pi G}{c \phi} \frac{d \tau}{d s} \frac{\rho}{g^{1 / 2}} \frac{d \tau}{d t} .
$$

Note that unlike in standard GR, the Ricci scalar $R$ does not vanish when $\rho$ vanishes, because the KSF still contributes to $R$. This is also unlike the EM field, because the trace of $T_{\mu \nu}^{\mathrm{EM}}$ vanishes and does not contribute to $R$. The KSF behaves more like an expression of spacetime curvature than like an independent source of spacetime curvature, because it is a source of gravitational field deforming spacetime that is independent of $G$.

4.2. Electromagnetic Field Equations. Now we provide the modified Maxwell equations, with source terms, for the EM field:

$$
\nabla_{v}\left(\phi^{3} F^{v \mu}\right)=\frac{\rho}{g^{1 / 2}} \mu_{0} k c \frac{d x^{\mu}}{d t} \tilde{U}_{5}
$$

Note that the KSF behaves in the Maxwell equations (25) like a dielectric constant, whereas in the gravitational equations, it behaves like a variable gravitational constant-as expected from the field Lagrangian (5).

The current source in (25) reproduces the usual Maxwell source term, with the usual charge density $\sigma \longrightarrow \rho k c \tilde{U}_{5}$.

4.3. Scalar Field Equation. Now we provide the Kaluza scalar field equation, with sources

$$
\phi^{2}\left(\frac{3}{4} \phi^{2} k^{2} F_{\alpha \beta} F^{\alpha \beta}-R\right)=\frac{16 \pi G}{c^{3} \phi} \frac{d s}{d t} \frac{\rho}{g^{1 / 2}} \tilde{U}_{5}^{2} .
$$

This is a relatively new equation to physics, with no wellstudied analog outside the Kaluza theory.

In terms of electric and magnetic fields, $\mathbf{E}$ and $\mathbf{B}$, $F_{\alpha \beta} F^{\alpha \beta}=2\left(B^{2}-E^{2} / c^{2}\right)$.

The form (26) of the scalar field equation is algebraic in $\phi$. Yet $R$ depends on $\phi$ through (24), and so the algebraic field equation for $\phi$ has its own energy-momentum as a source. This means $\phi$ acts nonlinearly as its own source, very much like how gravitational energy-momentum acts nonlinearly as its own source. We already noted that $\phi$ energymomentum is not modulated by $G$ in the field equations (21), like other forms of mass-energy.

Equation (26) is new to physics for its source term, $\propto \tilde{U}_{5}^{2}$, which translates to quadratic in electric charge. Where the
Brans-Dicke scalar field finds its source in neutral matter, the KSF finds its source in electrically charged matter. Due to the quadratic source term, positively and negatively charged sources produce the same KSF. The KSF apparently coexists with electric fields, because both are produced by charged matter. Is the KSF weak enough that such a field could have gone undetected? Or is it just swamped by the strength of the Coulomb electric field? In fact, this question was addressed by Ref. [4], who found significant theoretical modifications to the Coulomb force. This question will be addressed in further detail in a subsequent work.

\section{Saturation Effects in the Field Equations}

We can now combine expressions (10), (16), and (17) for the Kaluza coupling coefficients with the preceding field equations. We find two distinct limits in the source terms, depending on the presence of charged matter and depending on the electric charge relative to the critical electric charge $Q_{\text {crit }}=m_{0} k c$.

5.1. Fields of Weakly Charged Sources, $Q \ll m_{0} k c$. In the limit of low specific charge, $Q \ll m_{0} k c$, then $d \tau / d s \simeq a / c$ and $U_{5} \simeq c \tilde{U}_{5} / a \phi^{2}$. Under the identification of charge (15), the Maxwell equations have their standard scalar-electromagnetic form irrespective of the magnitude of $\tilde{U}_{5}$ :

$$
\nabla_{v}\left(\phi^{3} F^{v \mu}\right)=\mu_{0} \frac{\sigma}{g^{1 / 2}} \frac{d x^{\mu}}{d t}=\mu_{0} J^{\mu}
$$

The gravitational field equations assume their standard scalar-tensor form in this limit:

$$
\begin{aligned}
G_{\mu \nu} \simeq & \phi^{-1} T_{\mu \nu}^{\phi}+\frac{8 \pi G}{\mu_{0} c^{4}} \phi^{2} T_{\mu \nu}^{E M} \\
& +\frac{8 \pi G}{c^{4} \phi} \frac{a \rho}{g^{1 / 2}} g_{\mu \alpha} \frac{d x^{\alpha}}{d t} g_{\nu \beta} \frac{d x^{\beta}}{d \tau}
\end{aligned}
$$

The scalar field equation assumes the form

$$
\frac{3}{2} \phi^{3} k^{2}\left(B^{2}-E^{2} / c^{2}\right)-3 \nabla_{\alpha} \nabla^{\alpha} \phi \simeq \frac{8 \pi G}{c^{2}} \frac{a \rho}{\phi g^{1 / 2}} \frac{d \tau}{d t}+\mu_{0} \frac{\sigma}{a \phi g^{1 / 2}} \frac{Q}{m_{0}} .
$$

It is quite interesting that the theory specifies separate sources in the KSF from charged particles and their electric fields.

5.2. Fields of Strongly Charged Sources, $Q \sim m_{0} k c$. In the limit of high specific charge, $Q \sim m_{0} k c$, we must use the full form of (10), (16), and (17).

Consider now the gravitational field equations (21) in this limit. As specific charge increases and $\tilde{U}_{5} \longrightarrow a$, the Kaluza coupling coefficient $(10) d \tau / d s \longrightarrow 0$, and it acts to neutralize the gravitational effect of the source, so that the matter term goes to zero in the gravitational field equations.

This is not a new effect to the Kaluza theory. It is rather the same sort of antigravitational effect of electrostatic energy 
discussed by Ref. [9] and seen in the Reissner-Nordstrom metric. For the gravitational effect of a highly charged particle to go to zero is an ADM mass effect that we might have expected from standard 4D theory alone.

The Maxwell equations (25) are invariant with respect to the magnitude of $\tilde{U}_{5}$ and have the same form as in (27).

Consider now the high-charge limit for the KSF (26). Now the value of $d s / d t \propto d s / d \tau$ in (16) is driven to a large value, depending on the magnitude of $a$. Furthermore, the variation is inversely proportional to $\tilde{U}_{5}$, implying that the scalar charge saturates and becomes linear in electric charge at high charge values.

\section{Discussion and Conclusions}

This work has considered 5D covariance requirements to construct a 5D energy-momentum source term for the $5 \mathrm{D}$ Einstein equations (1). The energy-momentum tensor and associated matter Lagrangian (20) were established. Combined with the field Lagrangian (5) established by Ref. [10], a complete Lagrangian specification of the unique Kaluza classical Kaluza theory is now provided.

A proper form for the energy-momentum tensor is important for establishing the correct modifications to the electromagnetic and gravitational field equations. In addition to the effects of a scalar field expected from other scalartensor or scalar-electromagnetic theories, a peculiar Kaluza coupling coefficient (10) arises for charged matter which acts to vary the source strengths in ways unknown to conventional physics.

The peculiar effects at high specific charge include a neutralization of gravitational mass-energy, and a saturation in scalar field sources in a way that goes from quadratic in electric charge to linear in electric charge. Previous authors have calculated significant modifications of the Lorentz force law [4], and some of the new physical effects described here may be detectable in ordinary electrical laboratories.

Finally, we note that the saturation effects discussed above depend on the identification of electric charge (15) and on the value fixed for $a$ in (8). An alteration of these parameters can shift the saturation effects onto the Maxwell equations, but they cannot be made to disappear from the theory altogether. The proper assignment of electric charge and $a$, and therefore isolation of the saturation effects, should be constrained by correspondence with $4 \mathrm{D}$ physics. This will be addressed in a subsequent work.

\section{Data Availability}

This is a theory paper, that does not reply on empirical data.

\section{Conflicts of Interest}

The author declares no conflicts of interest.

\section{Acknowledgments}

This work is supported by DARPA DSO under the award AQD number D19AC00020.

\section{References}

[1] T. Kaluza, "On the unity problem of physics," Sitzungsberichte der K. Preussischen Akademie der Wissenschaften zu Berlin, p. 966, 1921.

[2] P. G. Bergmann, "Unified field theory with fifteen field variables," Annals of Mathematics, vol. 49, no. 1, p. 255, 1948.

[3] M. Y. Thiry, Geometry - the equations of Kaluza's unified theory, Comptes rendus de l'Académie des Sciences, Paris, 1948.

[4] J. A. Ferrari, "On an approximate solution for a charged object and the experimental evidence for the Kaluza-Klein theory," General Relativity and Gravitation, vol. 21, no. 7, pp. 683695, 1989.

[5] R. Coquereaux and G. Esposito-Farese, "The theory of KaluzaKlein-Jordan-Thiry revisited," Annales de l'IHP Physique théorique, vol. 52, pp. 113-150, 1990.

[6] J. M. Overduin and P. S. Wesson, "Kaluza-Klein gravity," Physics Reports, vol. 283, no. 5-6, pp. 303-378, 1997.

[7] H. Goenner, "Some remarks on the genesis of scalar tensor theories," General Relativity and Gravitation, vol. 44, no. 8, pp. 2077-2097, 2012.

[8] O. Klein, "The atomicity of electricity as a quantum theory law," Nature, vol. 118, no. 2971, p. 516, 1926.

[9] R. Arnowitt, S. Deser, and C. W. Misner, "Gravitationalelectromagnetic coupling and the classical self-energy problem," Physical Review, vol. 120, no. 1, pp. 313-320, 1960.

[10] L. L. Williams, "Field equations and Lagrangian for the Kaluza metric evaluated with tensor algebra software," Journal of Gravity, vol. 2015, Article ID 901870, 6 pages, 2015.

[11] C. Brans and R. H. Dicke, "Mach's principle and a relativistic theory of gravitation," Physical Review, vol. 124, no. 3, pp. 925-935, 1961.

[12] S. Carroll, Spacetime and geometry, Addison-Wesley, San Francisco, 2004.

[13] J. D. Bekenstein, "Fine-structure constant: is it really a constant?," Physical Review D, vol. 25, no. 6, pp. 1527-1539, 1982.

[14] J. Gegenberg and G. Kunstatter, "The motion of charged particles in Kaluza-Klein space-time," Physics Letters A, vol. 106, no. 9, pp. 410-414, 1984.

[15] J. D. Jackson, Classical electrodynamics, John Wiley \& Sons, New York, 2nd edition, 1975.

[16] S. Weinberg, Gravitation and cosmology, John Wiley \& Sons, New York, 1972. 